\title{
Extracting Impurity Locations using Scanning Capacitance Microscopy Measurements
}

\author{
Samira AGHAEI ${ }^{1}$, Petru ANDREI ${ }^{1}$, Mark HAGMANN ${ }^{2}$ \\ ${ }^{1}$ Department of Electrical and Computer Engineering, Florida State University, \\ Tallahassee, FL 32312, USA \\ ${ }^{2}$ Department of Electrical and Computer Engineering, University of Utah, \\ Salt Lake City, UT 84112, USA \\ sa13aa@my.fsu.edu
}

\begin{abstract}
In this article we investigate the possibility to use scanning capacitance microscopy (SCM) for the 2-D and 3-D "atomistic" dopant profiling of semiconductor materials. For this purpose, we first analyze the effects of random dopant fluctuations (RDF) on SCM measurements with nanoscale probes and show that the discrete and random locations of dopant impurities significantly affect the differential capacitance measured in SCM experiments if the dimension of the probe is below $50 \mathrm{~nm}$. Then, we present an algorithm to compute the $x, y$, and $z$ coordinates of the ionized impurities in the semiconductor material using a set of SCM measurements. The algorithm is based on evaluating the doping sensitivity functions of the differential capacitance and uses a gradientbased iterative method to compute the locations of dopants. Finally, we discuss a standard simulation case and show that we are able to successfully retrieve the locations of the ionized impurities using the proposed algorithm.
\end{abstract}

Index Terms-doping, fluctuations, ion implantation, nanoscale devices, scanning probe microscopy.

\section{INTRODUCTION}

Scanning capacitance microscopy (SCM) is one of the most commonly used techniques for dopant profiling in the semiconductor industry [1-4]. SCM uses a metalized probe tip to bias the semiconductor in the depletion mode and to measure the differential capacitance between the probe and the semiconductor material as a function of the bias voltage [5-9] (see Fig. 1). Since the differential capacitance is directly related to dopant and carrier concentrations, the capacitance-voltage $(\mathrm{C}-\mathrm{V})$ curves can be used to generate a 2D image of the carrier density near the surface of the semiconductor.

In this article we investigate the possibility to use SCM for 2-D and 3-D "atomistic" dopant profiling of semiconductor materials. As far as we know, this is the first attempt to use SCM to compute the exact locations of the dopant atoms in semiconductor materials. Previous works in the literature have focused on finding the average carrier distribution and dopant concentration inside the semiconductor, without giving any information about the individual location of the discrete ionized impurities inside semiconductor material.

The article is structured as follows. In the next section we present examples of RDF effects on the $\mathrm{C}-\mathrm{V}$ characteristics of metal probe-oxide-semiconductor structures. Then, we define the doping sensitivity functions that stay at the basis of our numerical algorithm for extracting the exact $(\mathrm{x}, \mathrm{y}, \mathrm{z})$ coordinates of ionized impurities in the semiconductor material. The algorithm uses the doping sensitivity functions of the differential capacitance and uses a gradient-based iterative method to compute the locations of dopants from the experimental $\mathrm{C}-\mathrm{V}$ curves. In section IV we summarize the numerical algorithm and in section $\mathrm{V}$ present numerical results for a few standard cases. Conclusions are presented in section VI.

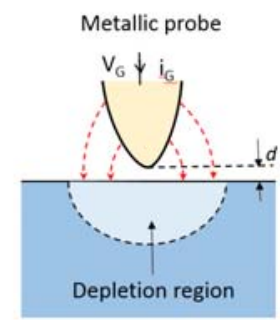

(a)

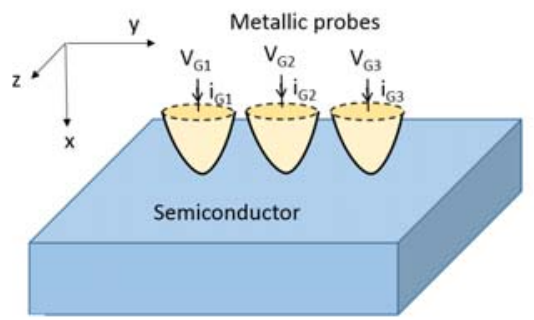

(b)
Figure 1. Traditional SCM setup with one probe (a). SCM setup with three metallic probes (b) used in this work.

\section{RDF EFFECTS ON THE C-V CHARACTERISTICS}

Most of the existing work related to the analysis of RDF effects in semiconductor devices focuses on the study of the variability of threshold voltages [10], current characteristics [11], and small-signal parameters [12] in field-effecttransistors and there are practically no studies related to the variability of $\mathrm{C}-\mathrm{V}$ curves besides a few recent conference presentations $[6,13,14]$. Next, we present a number of simulation studies that show the substantial effect that the discrete nature of dopant impurities has on the lowfrequency differential capacitance in SCM measurements. All the simulations presented in this work are performed by solving the Poisson equation coupled with the electron and hole continuity equations to describe the transport of the semiconductor, and with the density-gradient equations to describe quantum confinement effects at the semiconductor boundaries and near impurities. The complete system of equations was discretized using finite elements and solved in our in-house simulation software, RandFlux [15].

Fig. 2 shows the electrostatic potential, electron and hole concentrations in a atomistically doped silicon wafer, in which the metallic probe is situated at $1 \mathrm{~nm}$ above the surface of an n-type semiconductor and has a diameter between 10-15 $\mathrm{nm}$. The average doping concentration in the wafer is $10^{18} \mathrm{~cm}^{-3}$ and the potential of the probe with respect to flat bands in the semiconductor is shown in each figure. The dark regions in Figs. 2(a) and 2(b) denote regions of 
high electrostatic potential generated by individual dopant atoms. At the above value of the bias voltage the electrode creates a small depletion layer below the probe, which is visible in Figs. 2 (c) and Fig. 2(d). Although the depletion layer extends for a few tens of $\mathrm{nm}$ in the bulk of the semiconductor, the total number of dopants in this layer is relatively small (usually less than 10) and depends on the bias voltage [13]. (a)

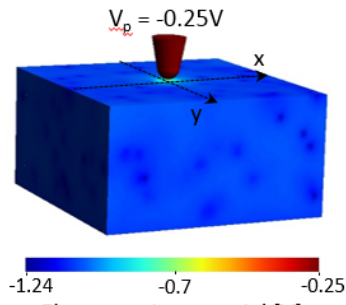

Electrostatic potential [V]

(c)

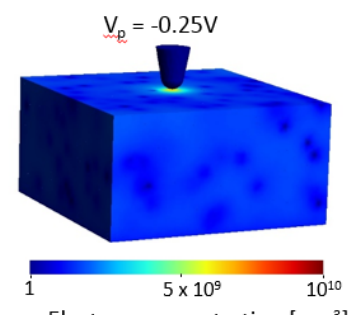

(b)

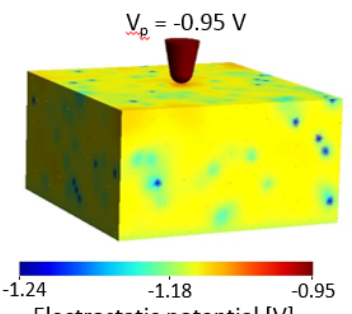

(d)

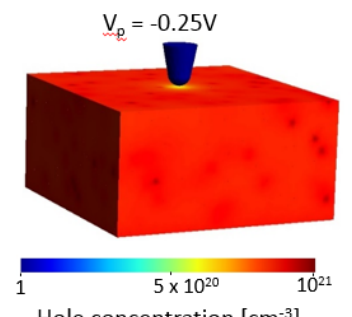

Figure 2.(a),(b) Electrostatic potential, (c) electron concentration, (d) and hole concentration in an n-type semiconductor at a bias voltage of $\mathrm{V}=-0.25 \mathrm{~V}$.

The simulated differential capacitance is presented in Figs. 3 for different probe diameters. Each figure presents a set of $200 \mathrm{C}-\mathrm{V}$ curves measured at different locations on the surface of the semiconductor material. The average doping concentration and the distance between the metallic probe and the semiconductor is the same as in the simulations presented in Fig. 2. The insets in these figures show the electrostatic potential computed at a particular location by emphasizing the size of the metallic probe used in simulations. The thicker black curve denotes the $\mathrm{C}-\mathrm{V}$ that would be obtained if the dopant concentration was a continuous function of the position inside the semiconductor (as it is usually done in numerical simulations).

It is important to notice that the size of the probe changes both the magnitude and the standard deviation of the differential capacitance. Hence, if we would like to use such measurement to obtain information about the exact location of ionized impurities we need to carefully select the size of the metallic probe. It is apparent from these figures that, on one hand, smaller probe sizes can distinguish better the different dopant configurations and result in a wider distribution of the $\mathrm{C}-\mathrm{V}$ curves. On the other side, the magnitude of the differential capacitance decreases when the probe size is decreasing leading to larger signal-to-noise ratio, which makes $\mathrm{C}-\mathrm{V}$ measurements prone to experimental errors.

\section{DOPING SENSITIVITY FUNCTIONS}

The inversion algorithm used in this article is based on the evaluation of the doping sensitivity functions of the differential capacitance. The doping sensitivity functions were originally introduced in $[16,17]$ to show how much the threshold voltage or the terminal currents of a metal-oxide-

semiconductor transistor are changing by adding one dopant atom at a given location inside the transistor.
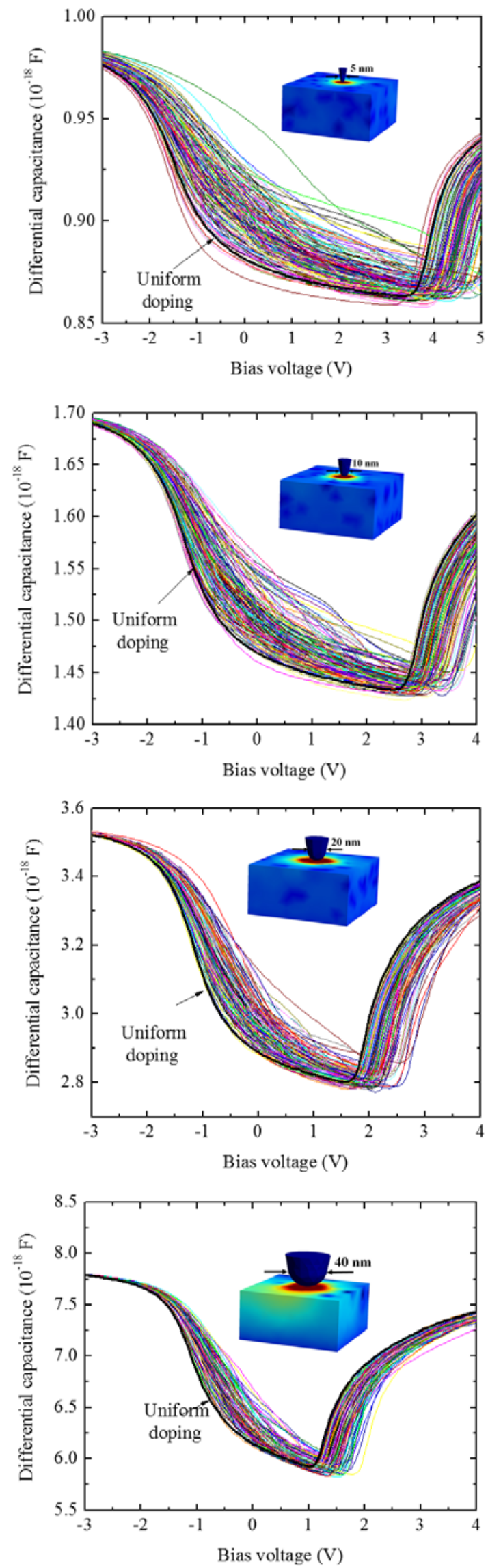

Figure 3. C-V characteristics measured at different locations on a semiconductor with five different probe sizes $(5 \mathrm{~nm}, 10 \mathrm{~nm}, 20 \mathrm{~nm}$ and 40 $\mathrm{nm})$ with average dopant concentration of $10^{18} \mathrm{~cm}^{-3}$.

They were later implemented in commercial device 
simulators [18] and generalized to describe the doping sensitivity of any device parameter such as small-signal parameters or subthreshold voltage swing [12]. Unlike in our previous works, in this section we follow Cacuci [19] and present an alternative definition of the sensitivity function in terms of the Gâtaux differential. Notice that the definition of the sensitivity functions is relatively general and is not related to any particular transport model.

Let us denote the doping concentration by $N_{0}(\boldsymbol{r})$; in large semiconductor devices, function $N_{0}(\boldsymbol{r})$ can be approximated as a continuous function, however, at nanoscale dimensions, the doping concentration can be written as a superposition of Dirac- $\delta$ functions. We also assume that the semiconductor material occupies a volume $\Omega$, which is a subset of the $J$-dimensional real vector space $R^{J}$, where $J=1,2$, or 3 . The position vector is denoted by $r \in \Omega$. For the purpose of this presentation we also define the following quantities:

1) $\boldsymbol{x}(\boldsymbol{r})=\left[x_{1}(\boldsymbol{r}), \ldots, x_{K}(\boldsymbol{r})\right]$ is the vector of state variables; $\boldsymbol{x} \in E_{x}$, where $E_{x}$ is a normed linear space over the scalar field of real numbers. If we use the densitygradient model, the components of $\boldsymbol{x}$ are the electrostatic potential, the electron and hole concentrations, the electron and hole quasi-Fermi potentials, lattice temperature, and the values of the current and potentials in the circuit network.

2) $\boldsymbol{N}(\boldsymbol{r})=\left[N_{1}(\boldsymbol{r}), \ldots, N_{I}(\boldsymbol{r})\right]$ is the doping concentration vector, which consists of the acceptor and donor doping concentrations as well as of any other ionized or neural impurities existent in the semiconductor. In general, $\boldsymbol{N}(\boldsymbol{r}) \in E_{N}$, where $E_{N}$ is also a normed linear space, which, in general is a subset of real scalars (i.e. $\left.E_{N} \subset R^{I}\right)$.

3) $\boldsymbol{F}(\boldsymbol{x}, \boldsymbol{N})=\left[F_{1}(\boldsymbol{x}, \boldsymbol{N}), \ldots, F_{K}(\boldsymbol{x}, \boldsymbol{N})\right]$ are in general $K$ nonlinear operators, including differential differences, integrals, distributions, or matrices) of $\boldsymbol{x}$ and $\boldsymbol{N}$. This operator is given by the particular transport system used to compute the state variables. This system is be written as:

$$
\boldsymbol{F}[\boldsymbol{x}(\boldsymbol{r}), \boldsymbol{N}(\boldsymbol{r})]=0, r \in \Omega
$$

In the framework of the drift-diffusion or density-gradient model $\boldsymbol{F}$ is a differential operator, however, in the framework of the Boltzmann transport equation $\boldsymbol{F}$ becomes an integro-differential operator.

4) $C(\boldsymbol{x})$ is the system response (i.e. the differential capacitance).

For any given doping concentration $N_{0}$, the state variables can be computed by solving the following nonlinear problem:

$$
\boldsymbol{F}\left[\boldsymbol{x}_{0}, \boldsymbol{N}_{0}\right]=0
$$

and the differential capacitance is $C\left(\boldsymbol{x}_{0}, \boldsymbol{N}_{0}\right)$. Suppose now that there is a small variation in the values of doping concentration $N_{0}+\Delta \boldsymbol{N}$. The variation in the values of the state variables can be computed by solving:

$$
F\left[x_{0}+\Delta x, N_{0}+\Delta N\right]=0
$$

and the variation in the differential capacitance is $C\left[x_{0}+\Delta x, N_{0}+\Delta N\right]-C\left(x_{0}, N_{0}\right)$. The Gâteaux derivative of the differential capacitance in the direction of variation $\Delta \boldsymbol{N}$ can be written as:

$$
\begin{gathered}
\gamma_{C}^{N_{0}}(\Delta \boldsymbol{N})=\lim _{\omega \rightarrow 0} \frac{C\left[\boldsymbol{x}_{0}+\alpha \Delta \boldsymbol{x}, \boldsymbol{N}_{0}+\alpha \Delta \boldsymbol{N}\right]-C\left(\boldsymbol{x}_{0}, \boldsymbol{N}_{0}\right)}{\alpha} \\
=\left\{\frac{d}{d \alpha} C\left[\boldsymbol{x}_{0}+\alpha \Delta \boldsymbol{x}, \boldsymbol{N}_{0}+\alpha \Delta \boldsymbol{N}\right]\right\}_{\alpha=0}
\end{gathered}
$$

For infinitesimally small variations $\Delta \boldsymbol{N}$, the Gâteaux derivative of the differential capacitance can be related to the doping sensitivity function of the differential capacitance $\gamma_{C}(\boldsymbol{r})$ using the Riesz representation theorem (see [17]):

$$
\gamma_{C}^{N_{0}}(\delta \boldsymbol{N})=\int_{\Omega} \gamma_{C}(\boldsymbol{r}) \delta \boldsymbol{N} d \boldsymbol{r}
$$

In the next section we focus our analysis on the numerical algorithm for the computation of the spatial coordinates of ionized impurities.

\section{NUMERICAL ALGORITHM}

The problem that we address in this section is how to compute the spatial coordinates of the ionized impurities inside the semiconductor material by using a set of $\mathrm{C}-\mathrm{V}$ curves measured experimentally. To solve this problem we need to develop an inversion algorithm that "extracts" the doping concentration from the experimental data. The numerical algorithm that we present below can be applied to any transport model that can be used to simulate the $\mathrm{C}-\mathrm{V}$ curves, such as the classical drift-diffusion model, the semiclassical Boltzmann equation, or the quantum NEGF method. We assume that the doping sensitivity functions of the differential capacitance can be computed by using the numerical techniques presented in references $[13,20]$.

Let us consider that we measure experimentally the differential capacitance as a function of the probe potential and we denote these data points by:

$$
C_{i}^{(\exp )}\left(V_{i}\right) \text {, where } i=1, \ldots, M
$$

where $V_{i}$ denote the potential of the probe at which measurement $i$ was made, $M$ is the number of data points, and index $i$ denotes the number of the measurement. Now, assume that we start with an initial doping profile $N_{0}(\boldsymbol{r})$ and compute numerically the differential capacitance $C$ at each potential $V_{i}$. Denote the computed values of the differential capacitance by:

$$
C_{i}^{(0)}\left(V_{i}\right) \text {, where } i=1, \ldots, M
$$

The inversion problem can now be formulated as follows. Given experimental data $C_{i}^{(\exp )}\left(V_{i}\right)$ and an initial profile of the doping concentration, how much do we need to change the doping concentration in order for the simulated value of the differential capacitance to fit the experimental data as closely as possible? Let us denote the solution to this problem by $\Delta N(r)=N(r)-N_{0}(r)$ and the required change in the value of the differential capacitance by $\Delta C_{i}=C_{i}^{(\text {exp })}-C_{i}^{(0)}$. According to the definition of the sensitivity function, we can write, $\delta C_{i}=\int_{\Omega} \gamma_{i}(\boldsymbol{r}) \delta N(\boldsymbol{r}) d \boldsymbol{r}$, which can be approximated as: 


$$
\Delta C_{i} \approx \int_{\Omega} \gamma_{i}(\boldsymbol{r}) \Delta N(\boldsymbol{r}) d \boldsymbol{r}
$$

Hence, if the initial guess of the doping concentration is not too far away from the real one, the last integral equation can be solved to compute $\Delta N(\boldsymbol{r})$. To solve this equation we discretize the integral numerically to get:

$$
\sum_{j=1}^{L} \gamma_{i}\left(r_{j}\right) \Delta N\left(r_{j}\right) \approx \Delta C_{i}
$$

where index $j$ denotes the location of the nodes in the finite element discretization and $L$ denotes the number of nodes. If the sensitivity functions are linearly independent, the integral equation (8) can be solved to compute $\Delta N(\boldsymbol{r})$. In discretized form, if matrix $\gamma_{i}\left(r_{j}\right)$ is not singular, and $L \leq M$, system (9) can be solved numerically in the least square sense to compute $\Delta N\left(r_{j}\right)$.

However, there are two major deficiencies with the above procedure. First, the number of $\mathrm{C}-\mathrm{V}$ measurements is of the order of $M=100$ 's and is usually much smaller than the number of discretization mesh points, which is the order of $L=10^{6}$ in 3-D problems. Hence, system (9) does not have unique solution. Second, if the number of C-V measurements is large enough, equation (9) represents a dense system of equations which is difficult to solve on normal computing systems. Hence, it is more convenient to expand the doping concentration $\Delta N(\boldsymbol{r})$ as a linear superposition of some basis functions $\Gamma_{k}(\boldsymbol{r})$ :

$$
\Delta N(\boldsymbol{r})=\sum_{k=1}^{K} \alpha_{k} \Gamma_{k}(\boldsymbol{r})
$$

where $\alpha_{k}$ are $K$ superposition coefficients that need to be determined. To determine these coefficients we multiply (10) by $\gamma_{i}(\boldsymbol{r})$ and integrate over $\Omega$ :

$$
\int_{\Omega} \Delta N(\boldsymbol{r}) \gamma_{i}(\boldsymbol{r}) d \boldsymbol{r}=\sum_{k=1}^{K} \alpha_{k} \int_{\Omega} \Gamma_{k}(\boldsymbol{r}) \gamma_{i}(\boldsymbol{r}) d \boldsymbol{r}
$$

Denoting the scalar products by $\gamma_{i, j}$ :

$$
\gamma_{k, i}=\int_{\Omega} \Gamma_{k}(\boldsymbol{r}) \gamma_{i}(\boldsymbol{r}) d \boldsymbol{r}
$$

coefficients $\alpha_{k}$ can be determined by solving the following system of equations using linear least square approximations

$$
\begin{gathered}
\sum_{k=1}^{K} \alpha_{k} \gamma_{k, i}=\int_{\Omega} \Delta N(\boldsymbol{r}) \gamma_{i}(\boldsymbol{r}) d \boldsymbol{r} \\
\sum_{k=1}^{K} \alpha_{k} \gamma_{k, i}=\Delta C_{i}
\end{gathered}
$$

where we have used (4).

In order for system (14) to have unique solution, we need in general $K \leq M$. Ideally, basis functions $\Gamma_{k}(\boldsymbol{r})$ should be selected in such a way to form a complete set in the space of the possible dopant profiles. One such selection is to use:

$$
\Gamma_{k}(\boldsymbol{r})=\delta\left(\boldsymbol{r}-\boldsymbol{r}_{k}\right)
$$

where $\delta$ is the Dirac-delta function. This choice of the basis functions is preferable in nanoscale dopant profiling because it accounts for the discrete nature of the dopant impurities. In addition, these basis functions simplify the numerical implementation significantly. Indeed, equation becomes:

$$
\gamma_{k, i}=\int_{\Omega} \delta\left(\boldsymbol{r}-\boldsymbol{r}_{k}\right) \gamma_{i}(\boldsymbol{r}) d \boldsymbol{r}=\gamma_{i}\left(\boldsymbol{r}_{k}\right)
$$

and system (14) becomes:

$$
\sum_{k=1}^{K} \alpha_{k} \gamma_{i}\left(\boldsymbol{r}_{k}\right)=\Delta C_{i}
$$

Another choice for the basis function is to select $\Gamma_{k}(\boldsymbol{r})=\gamma_{k}(\boldsymbol{r})$. In this case we need to compute the superposition coefficients by solving system (14) where:

$$
\gamma_{k, i}=\int_{\Omega} \gamma_{k}(\boldsymbol{r}) \gamma_{i}(\boldsymbol{r}) d \boldsymbol{r}
$$

This choice of the basis functions is appropriate for the case when we need to compute the 3-D doping profile, where the doping concentration can be approximated as a continuous function (for instance in large devices).

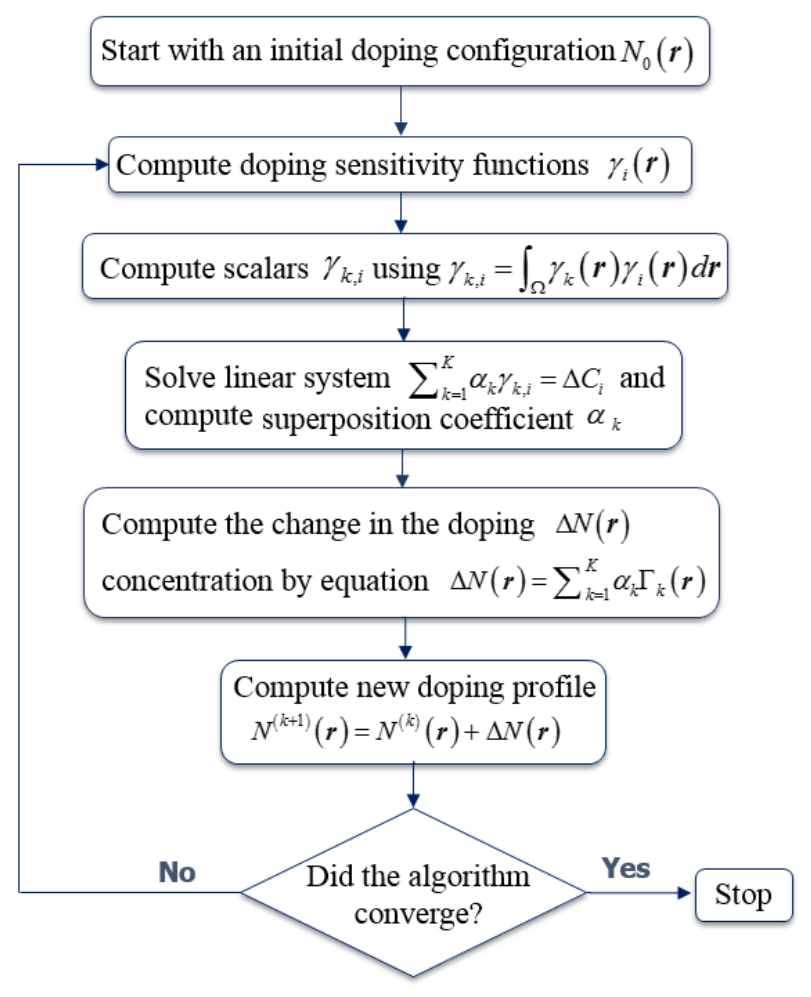

Figure 4. Numerical algorithm for the computation of doping profile in semiconductor devices.

\section{Simulation Results}

In this section we present a simulation case in which we use SCM to obtain the locations of the dopants in a 2-D semiconductor. For this purpose we use the atomistic doping profiling algorithm presented in previous section to extract the $(\mathrm{x}, \mathrm{y})$ locations of dopant atoms from a set of "experimental" capacitance-voltage (C-V) measurements. Since at this stage we are interested only in analyzing the feasibility of the numerical algorithm, the "experimental" data will be generated numerically in advance and, then, used to compute the doping profile.

In order to test the accuracy of our algorithm we use three identical metallic probes. The differential capacitance is measured while varying the bias voltage on each probe from $-3 \mathrm{~V}$ to $4 \mathrm{~V}$, while the other two probes are grounded. Notice that, to extract the doping profile (or spatial coordinates of dopants) in 2-D simulations, we need to use at least two metallic probes situated at two different locations on the semiconductor. In the case of 3-D semiconductors we need to use at least three metallic probes to extract the spatial coordinates of dopants inside the depletion region. 


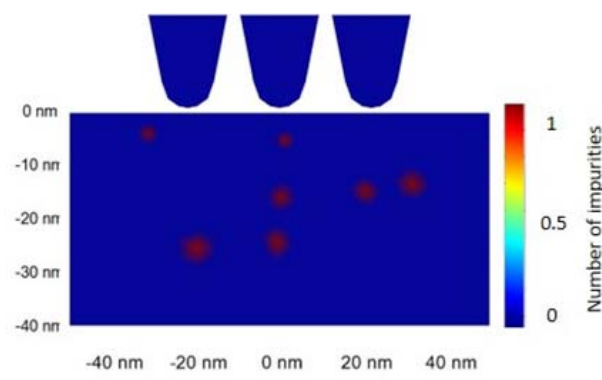

(a)

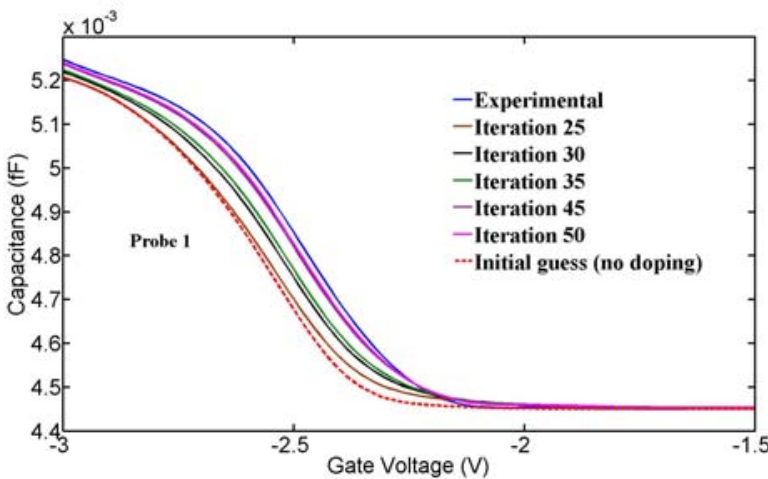

(b)

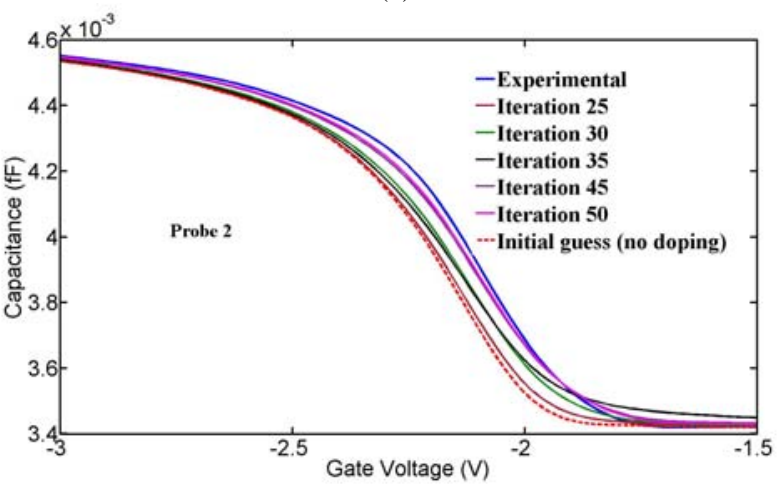

(c)

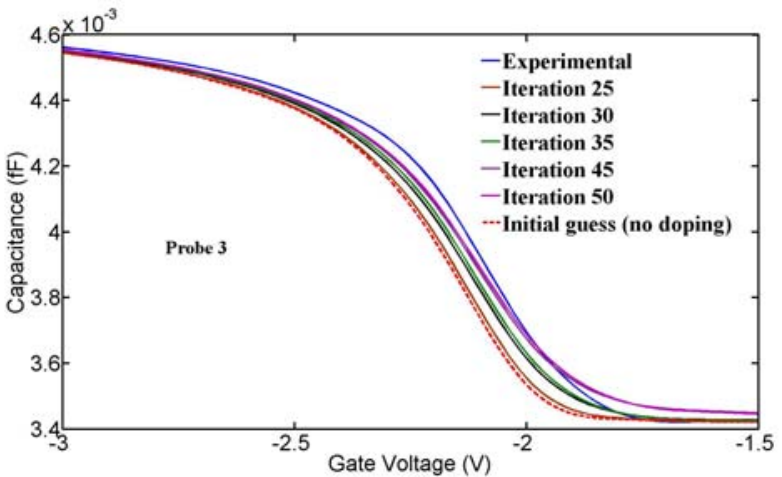

(d)

Figure 5. (a) Initial placement of impurities. (b)-(d) Differential capacitance as a function of bias voltage for each probe.

In order to test our algorithm, the "experimental" data is generated numerically as follows.

1) First, we build and mesh the system, including the metallic tips, vacuum, and semiconductor material.

2) Then, we simulate the ion implantation (or diffusion) process by placing randomly p-type impurities throughout the semiconductor material.

3) Then, we compute the a.c. current collected by each probe $i^{(k)}(\omega)$, under different bias conditions by solving the Poisson, current continuity, and density-gradient equations. The small-signal current is computed by superimposing a small-signal a.c. voltage, $v^{(k)}(\omega)$ on the d.c. bias.

4) Finally, we compute the differential capacitance using

$$
C^{(k)}(\omega)=\frac{i^{(k)}(\omega)}{v^{(k)}(\omega) j \omega}=\frac{1}{Z^{(k)}(\omega) j \omega}
$$

where $k=1,2$ and $3, j=\sqrt{-1}$, and $Z^{(k)}(\omega)$ is the complex impedance.

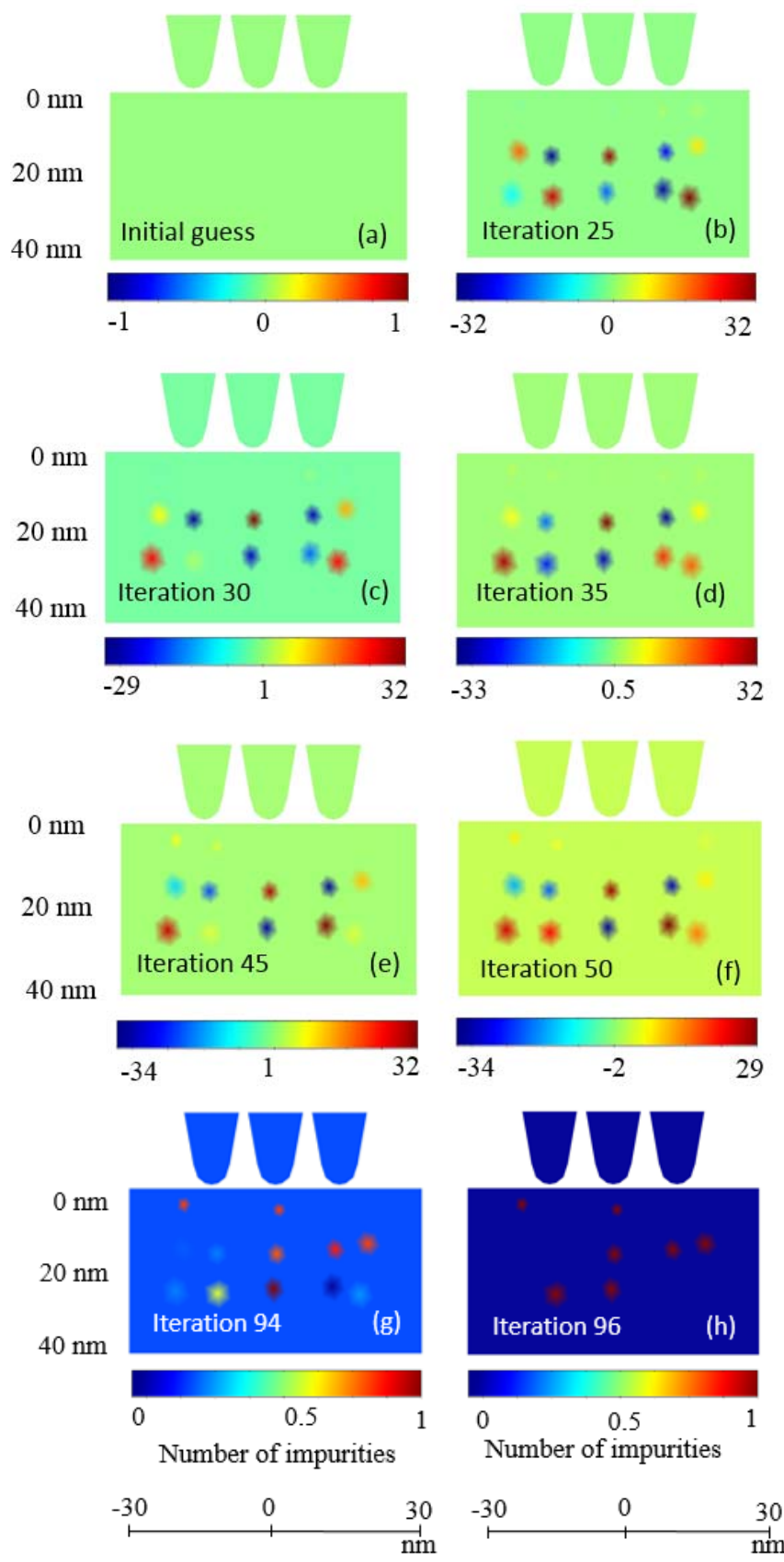

Figure 6. Predicted doping profile as a function of the iteration number. The algorithm started with and undoped structure (a) and converged to the original dopant distribution shown in (h).

The diameter of the tip of each probe is $10 \mathrm{~nm}$ and the probes are situated at a distance of $17.5 \mathrm{~nm}$ from each other. In a more practical setup one could consider only one metallic probe, which is allowed to move across the surface of the semiconductor so the $\mathrm{C}-\mathrm{V}$ curves would be collected 
at three different locations.

Fig. 5(a) presents the initial placement of the impurities, while Figs. 5(b)-(d) show the computed C-V curves as a function of the d.c. bias on each probe with continuous lines. In these simulations we consider 7 dopants randomly distributed in the semiconductor. The basis set is given by 15 Dirac-delta functions distributed approximately uniformly throughout the semiconductor. Notice that if a stochastic algorithm is used to simulate all the doping configurations, and if we assume each place can be occupied by at most one dopant atom, we need to perform $2^{15}=32,768$ simulations in order to find the optimum configuration.

The iterations start with an undoped material (see Fig. 6(a)) and the doping concentration is updated at each iteration using $N^{(k+1)}(\boldsymbol{r})=N^{(k)}(\boldsymbol{r})+\Delta N(\boldsymbol{r})$.

The computed differential capacitance at different iteration numbers is represented in Fig. 5 with continuous lines. The algorithm converged to the "experimental" values of the capacitance and the original doping profile is recovered exactly after 96 iterations as shown in Fig. 6(h). The total number of iterations depends on the initial guess, and the total number and microscopic distribution of impurities. The total simulation time depends on the number of mesh points and on the transport model used to compute the $\mathrm{C}-\mathrm{V}$ curves. In the framework of the drift-diffusion (coupled with the density-gradient equations) it takes a few minutes to retrieve the impurity locations for a mesh of 10,000 mesh points on a single processor computer.

\section{CONCLUSION}

We have shown that SCM measurements can be used to extract the 2-D spatial coordinates of ionized impurities in semiconductor devices. The proposed algorithm requires a few sets of $\mathrm{C}-\mathrm{V}$ curves measured at different locations on the surface of the semiconductor material and can be naturally extended to 3-D dopant profiling. Future work will include improving the convergence of the numerical algorithm in the presence of experimental noise and measurement errors and decreasing the total number of iterations.

\section{REFERENCES}

[1] L. Kosbar, J. Nxumalo, J. Nalaskowski, L. Hupka, C. Molella, J. Liu, G. Totir, K. Fisher, J. Cotte and M. Hopstaken, "Evaluation of emitter profiles and lateral uniformity on crystalline silicon photovoltaic cells using scanning capacitance microscopy," in Proc. 35th IEEE Photovoltaic Specialists Conference (PVSC), Yorktown Heights, New York, 2010, pp. 001737-001741. doi:10.1109/PVSC.2010.5616136

[2] H.S. Choi, Y.W. Han and I.S. Chung, "MOSFET implant failure analysis using plane-view scanning capacitance microscopy coupled with nano-probing and TCAD modeling," in Proc. 21th IEEE International Symposium on the Physical and Failure Analysis of Integrated Circuits (IPFA), Marina Bay Sands, Singapore, 2014, pp. 5-8. doi:10.1019/IPFA.2014.6898128

[3] J.M. Yang, U. Shaislamov, M.S. Hyun, J.H. Yoo, J.W. Kim, N.Y. Kwak, W. Kim and J.K. Park, "Precise comparison of twodimensional dopant profiles measured by electron holography and scanning capacitance microscopy,” in Proc. IEEE Nanotechnology Materials and Devices Conference (NMDC), Jeju, 2011, pp. 240-243. doi:10.1019/NMDC.2011.6155352

[4] A.C. Keow, I. Bin Hashim and L.N. Sern, "Electrical fault localization and Scanning Capacitance Microscopy (SCM) analysis methodology on high RDSON failure of smart power technology IC device,” in Proc. 20th IEEE International Symposium on the Physical and Failure Analysis of Integrated Circuits (IPFA), Suzhou, China, 2013, pp. 423-426. doi:10.1109/IPFA.2013.6599193
[5] N. Rodriguez, J. Adrian, C. Grosjean, G. Haller, C. Girardeaux and A. Portavoce, "Evaluation of scanning capacitance microscopy sample preparation by focused ion beam," in Proc. 17th European Symposium on Reliability of Electron Devices, Failure Physics and Analysis, Wuppertal, Germany, 2006, pp. 1554-1557. doi:10.1016/j.microrel.2006.07.019

[6] M.J. Hagmann, P. Andrei, S. Pandey and A. Nahata, "Scanning frequency comb microscopy (SFCM): A new method showing promise for high-resolution carrier profiling in semiconductors,” in Proc. 25th Advanced Semiconductor Manufacturing Conference (ASMC), Saratoga Springs, New York, 2014, pp. 213-218. doi:10.1109/ASMC.2014.6847001

[7] L.S. Shyuan, K.G.C. Siong, L.N. Sern, "SCM application in localized 2D dopant profiling,” in Proc. 20th IEEE International Symposium on Physical and Failure Analysis of Integrated Circuits (IPFA), Suzhou, China, 2013, pp. 615-619. doi:10.1109/IPFA.2013.6599236

[8] G. Bartolucci, G. M. Sardi, R. Marcelli and E. Proietti, “Analytical evaluation of the capacitance of a conical sensor for micro-nano imaging techniques,” in Proc. 6th IEEE International Workshop on Advances in Sensors and Interfaces (IWASI), Gallipoli, Turkey, 2015, pp. 283-287, doi:10.1109/IWASI.2015.7184940

[9] S. P. Hong, Z.X. Hua, C.K Chung and A. Chin, "Application of scanning capacitance microscopy on SOI wafer in die-level failure analysis" in Proc. 21th IEEE International Symposium on the Physical and Failure Analysis of Integrated Circuits (IPFA), Marina Bay Sands, Singapore, 2014, pp. 46-49, doi:10.1109/IPFA.2014.6898163

[10] A. Asenov, "Random dopant induced threshold voltage lowering and fluctuations in sub-0.1 \&mu m MOSFET's: A 3-D "atomistic" simulation study,” IEEE Trans. Electron Devices, vol. 45, pp. 25052513, Dec. 1998. doi:10.1109/16.735728

[11] B. Cheng, S. Roy, G. Roy, F. Adamu-Lema and A. Asenov, "Impact of Intrinsic parameter fluctuations in decanano MOSFETs on yield and functionality of SRAM cells,” Solid-State Electronics, vol. 49, pp. 740-746, May. 2005. doi:10.1016/j.sse.2004.09.005

[12] P. Andrei, I. Mayergoyz, "Analysis of random-dopant induced fluctuations of frequency characteristics of semiconductor devices," Journal of Applied Physics, vol. 93(8), pp. 4646-4652, Apr. 2003. doi:10.1063/1.1557786

[13] S. Aghaei, M. Mehta, P. Andrei, M. Hagmann, "Challenges and opportunities in atomistic dopant profiling using capacitance-voltage measurements," in Proc. 25th Annual SEMI Advanced Semiconductor Manufacturing Conference (ASMC), Saratoga $\begin{array}{lllr}\text { Springs, New } \quad \text { York, 2014, pp. } & \text { 19-21. }\end{array}$ doi:10.1109/ASMC.2014.6847009

[14] S. Aghaei, P. Andrei, M. Hagmann, “Atomistic dopant profiling using scanning capacitance microscopy," in Proc. IEEE Workshop on Microelectronics and Electron Devices (WMED), Boise, Idaho, 2015, pp. 1-5. doi:10.1109/WMED.2015.7093691

[15] RandFlux 0.5, User guide, Florida State University, http://www.eng.fsu.edu/ms/RandFlux

[16] I.D. Mayergoyz, P.Andrei, "Statistical analysis of semiconductor devices,” Journal of Applied Physics, vol. 90(6), pp. 3019-3029, June 2001, doi:10.1063/1.1390499

[17] P. Andrei, I. Mayergoyz, "Quantum mechanical effects on random oxide thickness and random doping induced fluctuations in ultrasmall semiconductor devices,” Journal of Applied Physics, vol. 94(11), pp. 7163-7172, Nov. 2003, doi:10.1063/1.1625084

[18] A. Wettstein, O. Penzin, E. Lyumkis and W. Fichtner, "Random dopant fluctuation modelling with the impedance field method," in Proc. of International Conference on Simulation of Semiconductor Processes and Devices (SISPAD), Boston, Massachusetts, 2003, pp. 91-94. doi:10.1109/SISPAD.2003.1233645

[19] D.G. Cacuci. Sensitivity \& Uncertainly Analysis. Chapman \& Hall/CRC, 2003.

[20] S. Aghaei, P. Andrei, and M. Hagmann, "Towards atomistic dopant profiling using SCM measurements,” Proc. of the 2015 International Conference on Semiconductor Processes and Devices, Washington D.C., 2015, pp. 401-404, doi:10.1109/SISPAD.2015.7292345 